\title{
Measuring longitudinal beam parameters in the low energy section of the Oak Ridge Spallation Neutron Source accelerator
}

\author{
A. Shishlo ${ }^{*}$ and A. Aleksandrov \\ Oak Ridge National Laboratory, P.O. Box 2008, Oak Ridge, Tennessee 37831-6461, USA \\ Yong Liu \\ KEK/J-PARC, 1-1 Oho, Tsukuba 305-0801, Ibaraki-Ken, Japan \\ Zhijun Wang \\ Institute of Modern Physics, CAS, N 509, Nanchang Road, Lanzhou 730000, China
}

(Received 19 February 2018; published 14 September 2018)

\begin{abstract}
Knowledge of the longitudinal beam parameters is important for understanding beam dynamics in hadron linacs. Recently, a noninterceptive method suitable for measuring the longitudinal rms Twiss parameters in the high energy part of a hadron linac was developed and experimentally verified at the Spallation Neutron Source (SNS). This work presents an extension and modification of the method to allow measurements in the low energy part of a linac. Like the original method, amplitude of the beam rf harmonic measured by the beam position monitor is used to derive the information on the bunch length. The new method was tested at the medium energy beam transfer line of the SNS accelerator. The results are in good agreement with an independent measurement of the bunch length using the longitudinal acceptance scan of the drift tube linac. The suggested method is fast, and it can be a useful part of the initial longitudinal beam tuning at the front end of hadron linacs.
\end{abstract}

DOI: 10.1103/PhysRevAccelBeams.21.092803

\section{INTRODUCTION}

Knowledge of longitudinal beam parameters in hadron linacs is important for understanding beam dynamics and, eventually, tuning the machine for low beam loss. It is notoriously difficult to measure the longitudinal beam parameters of nonrelativistic beams. A few years ago, a nonintercepting method for the longitudinal rms Twiss parameters measurements was developed for the superconducting part of the SNS accelerator [1].

The method is based on the analysis of the amplitude of the beam current Fourier harmonic during phase scan of the accelerating cavity. The amplitude of the harmonic is measured by the beam position and phase monitor (BPM), which sum signals are proportional to the amplitude of Fourier transformation of the longitudinal bunch distribution. Assuming a Gaussian longitudinal distribution, the rms bunch length can be found from the BPM amplitude signal

\footnotetext{
*shishlo@ornl.gov

Published by the American Physical Society under the terms of the Creative Commons Attribution 4.0 International license. Further distribution of this work must maintain attribution to the author(s) and the published article's title, journal citation, and DOI.
}

$$
\sigma^{2}=2 \cdot \ln \left(\frac{A_{\max }}{A}\right) .
$$

Here $\sigma, A, A_{\max }$ are the rms length of the bunch in radians, the BPM's amplitude and its maximal value respectively. During the phase scan of the first cavity of the SCL, all other cavities are switched off, and amplitude signals of all BPMs in the superconducting SNS linac are recorded as functions of the rf cavity phase. The switched off cavities give enough space for the bunch to grow longitudinally, so the BPMs will detect the change in the amplitude signals for different rf cavity phases. The method is an analog of a quadrupole field scan with the transverse profile measurements to find the transverse Twiss beam parameters, but instead of the quadrupole it uses the first rf cavity to transform the bunch in longitudinal phase space, and it uses BPMs instead of the profile monitor.

The important component of this method is a beambased calibration of the BPM amplitude signal. To find $A_{\max }$ in (1), the bunch length of the beam should be short relative to the period of the measured harmonic frequency. In the high energy part of the accelerator this is easily done by using the nominal linac rf settings when the bunch length is less than 2-3 degrees. At the low energy part of a hadron accelerator the nominal bunch length is typically not short, and the rf acceleration cavities are not powerful 
enough (at least this is true for the SNS accelerator) to compress the bunch to the length necessary for BPM amplitude calibration.

\section{EXTENSION OF THE ORIGINAL METHOD}

Inability to calibrate the absolute amplitude of the BPM signal using a short bunch measurement necessitates making the calibration coefficient an additional unknown parameter, which in turn requires adding more variable parameters to have a sufficient number of independent equations in the system. It turns out using a lattice with two rf cavities and one BPM,

$$
\text { rf Cavity + Drift + rf Cavity + Drift + BPM, }
$$

instead of only one rf cavity and BPM in the original method, is sufficient for determining the calibration coefficient and the Twiss parameters simultaneously. In the absence of collective effects like space charge, the initial and final longitudinal coordinates of the particles in the bunch satisfy the set of linear equations,

$$
\left[\begin{array}{c}
\varphi_{(i)} \\
E_{(i)}
\end{array}\right]=\left[\begin{array}{ll}
m_{1,1}^{(i)} & m_{1,2}^{(i)} \\
m_{2,1}^{(i)} & m_{2,2}^{(i)}
\end{array}\right] \cdot\left[\begin{array}{c}
\varphi_{\text {ini }} \\
E_{\text {ini }}
\end{array}\right], \quad i=1, N
$$

where $i$ is index of lattice states with different sets of rf cavities' phases and amplitudes, and $\varphi$ and $E$ are the longitudinal position and the kinetic energy deviations from the synchronous particle of the bunch. The transport matrices for rf cavities and drifts are defined as follows:

$$
\begin{aligned}
M_{\mathrm{rf}}\left(V_{0}, \phi_{\mathrm{rf}}\right) & =\left[\begin{array}{cc}
1 & 0 \\
-q V_{0} \sin \left(\phi_{\mathrm{rf}}\right) & 1
\end{array}\right], \\
M_{\text {Drift }}(L) & =\left[\begin{array}{cc}
1 & 2 \pi \frac{L}{\lambda_{\mathrm{rf}}} \frac{1}{m \gamma^{3} \beta^{3}} \\
0 & 1
\end{array}\right], \\
\lambda_{\mathrm{rf}} & =\frac{c}{f_{\mathrm{rf}}},
\end{aligned}
$$

where $q$ is the charge of the particles, $V_{0}, \phi_{\mathrm{rf}}, f_{\mathrm{rf}}$ are an effective voltage of the rf cavity, its phase and frequency; $L$ is a length of a drift, and $m, \gamma, \beta, c$ are mass, relativistic parameters of the particles, and the velocity of light respectively. The total transport matrix of the system (3) is

$$
\begin{aligned}
{\left[\begin{array}{ll}
m_{1,1}^{(i)} & m_{1,2}^{(i)} \\
m_{2,1}^{(i)} & m_{2,2}^{(i)}
\end{array}\right]=} & M_{\text {Drift }}(L 2) \times M_{\mathrm{rf} 2}\left(V_{02}^{(i)}, \phi_{\mathrm{rf} 2}^{(i)}\right) \\
& \times M_{\text {Drift }}(L 1) \times M_{\mathrm{rf} 1}\left(V_{01}^{(i)}, \phi_{\mathrm{rf} 1}^{(i)}\right) .
\end{aligned}
$$

Calculating the squares of both sides of the first equation in the system (3), and averaging over the whole ensemble of the particles, the squared rms bunch lengths at the end of the lattice are obtained:

$$
\begin{aligned}
\left\langle\varphi_{(i)}^{2}\right\rangle= & \sigma_{(i)}^{2} \\
= & \left(m_{1,1}^{(i)}\right)^{2} \cdot\left\langle\varphi_{i n i}^{2}\right\rangle+2 \cdot m_{1,1}^{(i)} \cdot m_{1,2}^{(i)} \cdot\left\langle\varphi_{i n i} \cdot E_{i n i}\right\rangle \\
& +\left(m_{1,2}^{(i)}\right)^{2} \cdot\left\langle E_{i n i}^{2}\right\rangle .
\end{aligned}
$$

After combining (1) and (6), it gives a set of linear equations with the measured amplitudes on the left side and four unknown parameters $\left\langle\varphi_{\text {ini }}^{2}\right\rangle,\left\langle\varphi_{\text {ini }} \cdot E_{\text {ini }}\right\rangle,\left\langle E_{\text {ini }}^{2}\right\rangle$, $2 \ln \left(A_{\max }\right)$ on the right side:

$$
\begin{aligned}
-2 \ln \left(A_{(i)}\right)= & \left(m_{1,1}^{(i)}\right)^{2} \cdot\left\langle\varphi_{\text {ini }}^{2}\right\rangle+2 \cdot m_{1,1}^{(i)} \cdot m_{1,2}^{(i)} \cdot\left\langle\varphi_{\text {ini }} \cdot E_{\text {ini }}\right\rangle \\
& +\left(m_{1,2}^{(i)}\right)^{2} \cdot\left\langle E_{\text {ini }}^{2}\right\rangle-2 \ln \left(A_{\text {max }}\right) .
\end{aligned}
$$

The elements of the transport matrix are defined by (4) and (5). The system (7) can be solved by using the least square method with the number of measurements $N \geq 4$. Increasing $N$ reduces the error of solution. The error analysis, and relations between initial statistical correlation in the bunch and Twiss parameters is described in the appendix of [1]. It should be noted that the system of equations (7) does not have solutions for a lattice with only one rf cavity and one drift like in the original method in [1].

\section{MEASUREMENT OF THE LONGITUDINAL TWISS PARAMETERS}

The algorithm described in the Sec. II was implemented using the two last rf cavities and the last BPM in the medium energy beam transfer (MEBT) section of the SNS accelerator [2]. The experimental hardware layout is shown schematically in Fig. 1. The $2.5 \mathrm{MeV} \mathrm{H}^{-}$beam from the radio frequency quadrupole (RFQ) is transported through the MEBT and matched transversely and longitudinally into the DTL. A beam current attenuation system consisting of a set of insertable semitransparent Tungsten grids allows the peak beam current to be reduced while preserving the transverse and longitudinal particle distribution. To reduce the effect of the space charge, the measurements described below were done using a $20 \%$ transmission grid that reduced the peak current from 30 to $6 \mathrm{~mA}$.

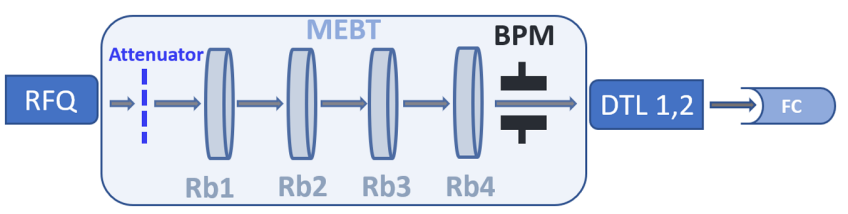

FIG. 1. A schematic layout of the RFQ, MEBT, and DTL 1,2 sections of the SNS linac. Only equipment relevant to the measurements is shown in the picture: a beam current attenuating grid, bunching rf cavities (Rb1, Rb2, Rb3, and $\mathrm{Rb} 4)$, a beam position and phase monitor (BPM), and a Faraday cup (FC). 
TABLE I. The longitudinal Twiss parameters at the Rb3 entrance.

\begin{tabular}{lcc}
\hline \hline$\alpha$ & $\beta, \operatorname{deg} / \mathrm{MeV}$ & $\varepsilon, \pi \operatorname{deg} \mathrm{MeV}$ \\
\hline$-0.723 \pm 0.035$ & $930 \pm 94$ & $0.23 \pm 0.01$ \\
\hline \hline
\end{tabular}

The rf cavity gradient and phase relative to the beam was calibrated using the standard MEBT rf setup procedure [3] before starting the longitudinal Twiss parameters determination scan.

Each measured point of the scan included the BPM amplitude, effective voltages for $\mathrm{Rb} 3$ and $\mathrm{Rb} 4$, and the $\mathrm{rf}$ phase of Rb4. The rf phase of Rb3 was kept constant at the bunching phase of -90 degrees. The full scan data set included seven effective voltages for the Rb3 (equidistant, from 28 to $69 \mathrm{kV}$ ), four effective voltages for the $\mathrm{Rb} 4$ (equidistant, from 51 to $103 \mathrm{kV}$ ), and 72 equidistant phases for the $\mathrm{Rb} 4$ (from $-180^{\circ}$ to $+180^{\circ}$ ). The total number of measurements and equations in the (7) system was $7 \times 4 \times 72=2016$. The measurements process was automated, and it took less than one hour with the beam repetition rate of $1 \mathrm{~Hz}$. The system of equations (7) was solved using the least square method (LSM). The matrix equations for the LSM are similar to ones described in the appendix of [1] with an increased number of variables from three to four. The found longitudinal Twiss parameters and errors at the entrance of the Rb3 rf cavity are shown in Table I. It should be noted that the measured longitudinal emittance is $80 \%$ above the design value. The measured Twiss parameters at the Rb3 entrance can be recalculated to any point in the MEBT using a combination of the transport matrices (4).

The good quality of agreement between the measured BPM amplitudes and the predicted by (7) values is demonstrated in Fig. 2 where one Rb4 phase scan result (one from the set of 28) is shown.

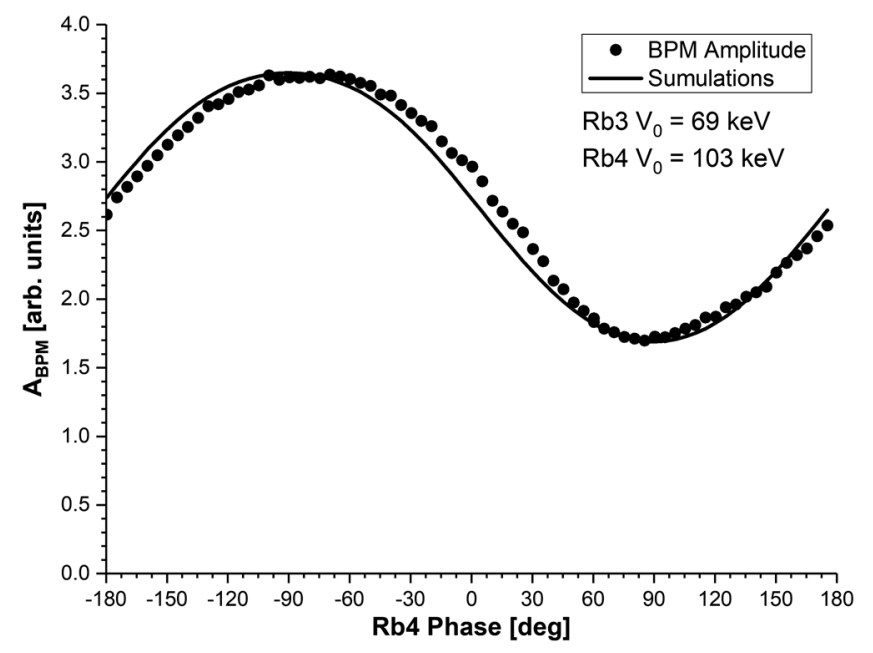

FIG. 2. The BPM amplitude vs Rb4 phase for one combination of the effective rf voltages.

\section{DTL ACCEPTANCE SCANS: BUNCH LENGTH VS RB4 EFFECTIVE VOLTAGE}

To confirm validity of the derived Twiss parameters the longitudinal bunch size was measured at the DTL entrance using the DTL longitudinal acceptance scans method. The DTL longitudinal acceptance scans are usually used for DTL tuning [4], but the data from the scans can be used to estimate the bunch length at the entrance of the DTL structure. During the scan the charge of the bunch delivered to the Faraday cup (FC in Fig. 1) is measured as a function of the simultaneous phase shift of DTL 1 and 2 tanks from the design rf phase. The FC is equipped with an energy filter which cuts out the nonaccelerated particles outside of the longitudinal acceptance. The scan process is schematically illustrated in Fig. 3. When the DTL synchronous phase is moving in the negative direction the bunch is moving to the right inside the DTL acceptance, more particles from the bunch are captured into the separatrix and reach the FC. The FC signal vs the phase shift curve is proportional to the integral of the bunch longitudinal distribution inside the separatrix.

The functional representation for the scan curve can be derived analytically assuming the Gaussian longitudinal density distribution:

$$
f(x)=\frac{1}{2}\left[1+\operatorname{erf}\left(\frac{x-x_{c}}{\sqrt{2} \cdot \sigma_{\phi}}\right)\right] .
$$

Here $x, x_{c}, \sigma_{\phi}$ are the phase shift from the design DTL rf phase, $50 \%$ transmission phase shift, and the rms bunch length respectively. An example of the transmission curve is shown in Fig. 4.

The bunch length parameter in formula (1) is only an approximation of the real bunch length. There are factors causing the difference between these two values. For example, the left border of the acceptance region in

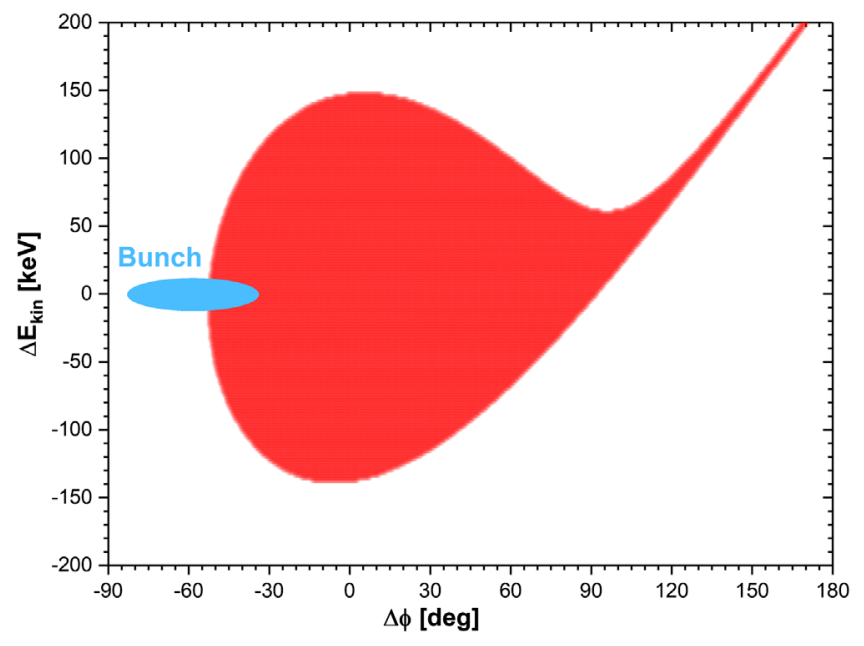

FIG. 3. DTL 1,2 longitudinal acceptance region. 


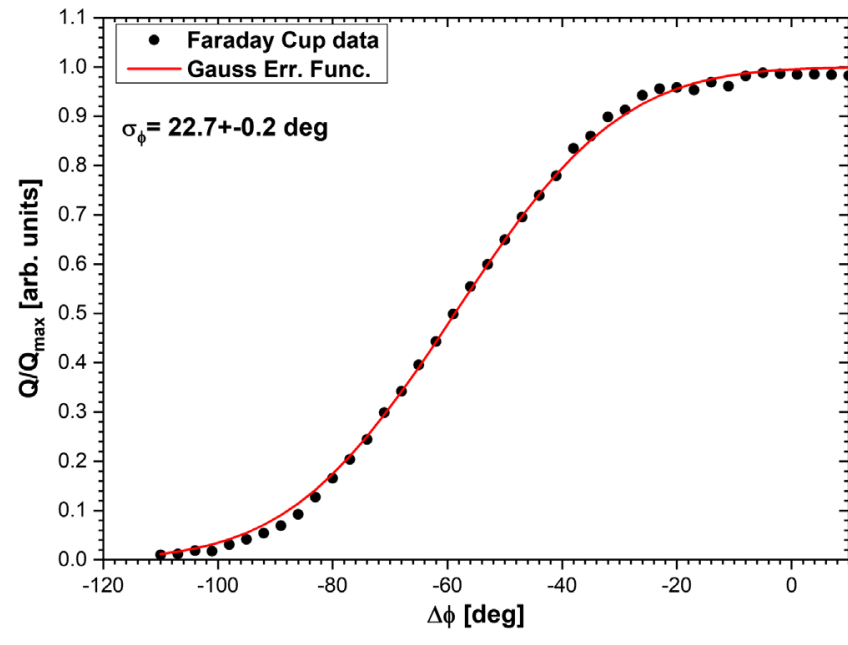

FIG. 4. The transmission curve of the DTL 1,2 acceptance scan and its approximation by the formula (1) for the Rb4 effective voltage $103 \mathrm{kV}$.

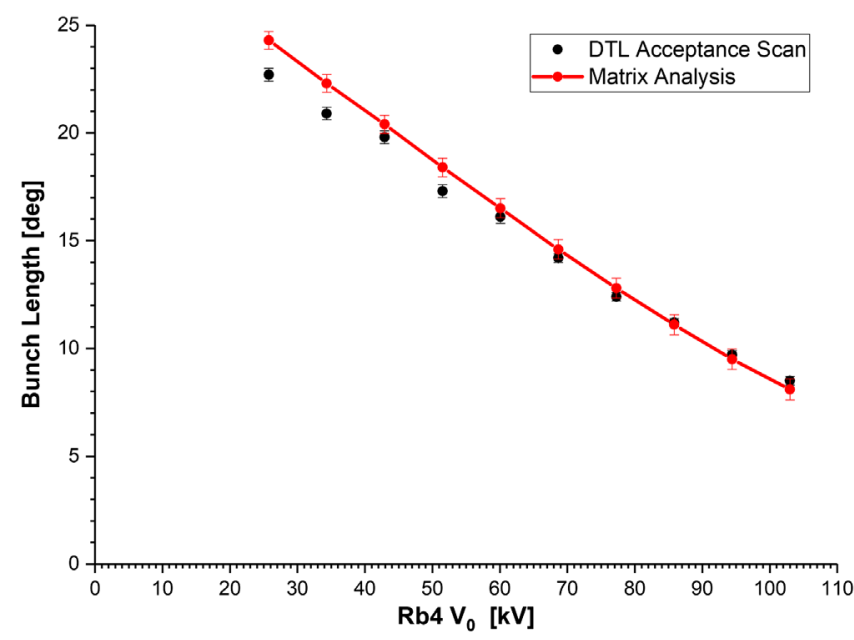

FIG. 5. The bunch length at the end of MEBT vs Rb4 voltage.

Fig. 3 is not a perfect vertical line, and the real ellipse describing the bunch in this phase space is tilted. Despite these approximations, the acceptance scans are widely used for the bunch length estimation $[5,6]$.

Figure 5 shows comparison between the bunch length values extracted from the DTL acceptance scans and the bunch lengths predicted using the measured Twiss parameters from Table I. Overall, Fig. 5 demonstrates good agreement between the two methods. Differences between the two methods' results become noticeable for relatively large bunch lengths, when the nonlinear corrections to the transformation through the rf gap (4) become significant.
It is possible to include into the analysis these nonlinear corrections by using realistic particle-in-cell simulation codes and general data fitting algorithms for the initial longitudinal Twiss parameters, but it is beyond the scope of the present paper.

\section{CONCLUSIONS}

In this work we described an extension and modification of the recently developed method [1] to measure the longitudinal Twiss parameters of a hadron beam using BPM signals. This modification allows measuring the Twiss parameters at the places where the beam-based amplitude calibration of the BPM cannot be performed. The modified method has been successfully tested at the low energy part of the SNS accelerator. The new method is relatively fast and can be easily automated.

\section{ACKNOWLEDGMENTS}

The work was performed at Spallation Neutron Source accelerator at Oak Ridge National Laboratory (ORNL). This manuscript has been authored by UT-Battelle, LLC, under Contract No. DE-AC05-00OR22725 with the U.S. Department of Energy.

[1] A. Shishlo and A. Aleksandrov, A non-interceptive method to measure longitudinal Twiss parameters of a beam in a hadron linear accelerator using beam position monitors, Phys. Rev. ST Accel. Beams 16, 062801 (2013).

[2] S. Henderson, W. Abraham, A. Aleksandrov et al., The Spallation Neutron Source accelerator system design, Nucl. Instrum. Methods Phys. Res., Sect. A 763, 610 (2014).

[3] A. Shishlo, A. Aleksandrov, A. Zhukov, and Yong Liu, Model based optics studies in the MEBT section of SNS, in Proceedings, 8th International Particle Accelerator Conference (IPAC 2017), Copenhagen, Denmark (CCBY-3.0, 2017), pp. 814-816.

[4] Edited by A. Chao, K. Mess, M. Tinger, and F. Zimmerman, Handbook of Accelerator Physics and Engineering, 2nd ed. (World Scientific, Singapore, 2013), p. 39.

[5] Y. Zhang, J. Galambos, and A. Shishlo, Measurement of longitudinal acceptance and emittance of the Oak Ridge Spallation Neutron Source Superconducting Linac, Phys. Rev. ST Accel. Beams 11, 104001 (2008).

[6] T. Maruta, A. Mira, H. Sako, G. Wei, and M. Ikegami, Longitudinal beam acceptance of J-PARC Drift Tube Linac, in Proceedings of the 2nd International Particle Accelerator Conference, San Sebastián, Spain (EPS-AG, Spain, 2011), pp. 2592-2594. 